\title{
The Potentiality of Human Umbilical Cord Isolated Mesenchymal Stem/Stromal Cells for Cardiomyocyte Generation
}

This article was published in the following Dove Press journal: Stem Cells and Cloning: Advances and Applications

\author{
Amoura Abou-EINaga' \\ Farha El-Chennawi ${ }^{2}$ \\ Samar Ibrahim kamel ${ }^{1}$ \\ Ghada Mutawa $\mathbb{D}^{3}$ \\ 'Zoology Department, Faculty of \\ Sciences, Mansoura University, Mansoura \\ 355 I6, Egypt; ${ }^{2}$ Clinical Pathology \\ Department, Faculty of Medicine, \\ Mansoura University, Mansoura 35516, \\ Egypt; ${ }^{3}$ Department of Basic Science, \\ Faculty of Dentistry, Horus University in \\ Egypt (HUE), New Damietta 345 I8, \\ Egypt
}

Background: The new therapeutic strategy of managing cardiac diseases is based on cell therapy; it highly suggests the use of multipotent mesenchymal stem/stromal cells (MSCs). MSCs widely used in researches are known to be isolated from bone marrow. However, this research seeks to use a human umbilical cord (HUC) as an alternative source of MSCs. Since HUC Wharton's jelly (WJ)-isolated MSCs originate as fetal tissue they are highly preferable for their potential advantages over other adult tissues.

Methods: The researchers used enzymatic digestion to establish a primary HUC-WJisolated MSC line. Then, flow cytometry was used to characterize MSCs and hematopoietic stem cells (HSCs) markers' expression. In addition, the cardiac differentiation capacity of HUC-WJ-isolated MSCs in vitro was investigated by two protocols. Protocol-1 necessitates the dependence on merely 5-azacytidine (5-Aza), whereas in protocol-2, 5-Aza was supported by basic fibroblast growth factor (BFGF). The comparative study between the two protocols was applied by inspecting the ultrastructure of differentiated cells, measuring RTPCR mRNA cardiac markers and the quantitative detection of cardiac proteins.

Results: HUC-WJ isolated MSCs were expressed by $\mathrm{CD} 90^{+\mathrm{ve}}, \mathrm{CD} 105^{+\mathrm{ve}}, \mathrm{CD} 106^{+\mathrm{ve}}$, CD45 ${ }^{-\mathrm{ve}}$, and CD146 ${ }^{-\mathrm{ve}}$. Remarkable TNNT1, NKX2.5, and Desmin mRNA expression and higher quantitative LDH and cTnI were detected by applying protocol-2. This same protocol-2 induced cardiac morphological features that were revealed by identifying cardiomyocyte-like cells and typical sarcomeres.

Conclusion: HUC-WJ is proved to be an ethical and effective source of MSCs induced cardiac differentiation, whereas BFGF supports 5-Aza in MSCs-cardiomyocytes differentiation.

Keywords: mesenchymal stem/stromal cells, human umbilical cord, Wharton's jelly, cardiomyocytes, 5-azacytidine, fibroblast growth factor

\section{Plain Language Summary}

Stem cell therapy has the ability to improve the traditional treatment of cardiac diseases by inducing stem cell differentiation into cardiomyocytes to repair damaged cardiac tissues. Many previous studies depended on the easy way that isolates stem cells from adult tissues like bone marrow and adipose tissue; however, the limited potentiality of such isolated stem cells delayed the progress of those studies. However, improving the potentiality of stem cells requires the development of applied protocols. So, this study was based on the human umbilical cord (HUC) Wharton's jelly (WJ) as a suitable source of mesenchymal stem/stromal cells (MSCs). HUC-WJ isolated MSCs have a differentiation potentiality into multiple cell types without any ethical concerns. The study also developed a new protocol by using 5-Azacytidine (5-Aza) and basic fibroblast growth factor (BFGF) to differentiate MSCs into cardiomyocytes. This protocol encourages cardiac diseases. The
Department of Basic Science, Faculty of Dentistry, Horus University in Egypt (HUE), International Coastal Road New, Damietta City, Kafr Saad, Damietta Governorate 34518, Egypt

Tel +201021888628

Fax +2-057-3286II

Email Ghada.adel.mutawa@gmail.com
Stem Cells and Cloning: Advances and Applications 2020:13 91-101 
resulting HUC-WJ isolated MSCs showed significant cardiac differentiation by acquiring special microscopic cardiac features, and by the high expression of cardiac markers. Accordingly, HUC-WJ isolated MSCs proved to be a promising strategy of applying stem cellbased therapy on cardiac diseases.

\section{Introduction}

Cardiac diseases have remained the leading cause of death all over the world. Over the past decades, the traditional treatment of cardiac diseases has resulted in a tragic rise in mortality rate as it just managed to temporarily delay the progress of the disease. ${ }^{1}$ Although remarkable evolution is noticed in the treatment of cardiac diseases, still there is a social and economic burden on health institutions. ${ }^{2}$ Therefore, the conventional treatment of cardiac diseases cannot provide an authentic cure.

The recent trend in regenerative medicine nowadays is using stem cells as recovering-cell therapy for cardiac diseases. Mesenchymal stem/stromal cells (MSCs) are the pioneer example of adult stem cells that can lead to the new regenerative strategy. ${ }^{3}$ MSCs are nonhematopoietic stem cells (HSCs), multipotent-adult stem cells as they can differentiate into all stromal connective linages like fat, cartilage, and bone cells. MSCs cannot be considered pluripotent-embryonic stem cells just as inner cell mass in blastula because they cannot differentiate into all human cell types. ${ }^{4}$ MSCs are characterized by specific markers called cluster of differentiation (CD), especially as CD90, CD105, and CD106. ${ }^{5}$ These markers trigger MSCs' unique stemness, growth, and differentiation properties. ${ }^{5}$ Also, MSCs lack markers of HSCs such as CD146 and CD45. ${ }^{5}$ MSCs have typical fibroblasticmorphology and adherent property to plastic surfaces during in vitro culture. All of these properties support the position of MSCs on applying regenerative therapy to solve the clashes in the treatment of cardiac diseases. ${ }^{2}$

MSCs are able to act as a repair-cellular therapy that is stimulated by as many pathological causes as the cardiac diseases. ${ }^{2}$ In the treatment of the cardiac diseases, there are many roles of MSCs in the mechanism of action, whereas they act as immune-regulators, anti-apoptotic, and allogeneic utilizers. Particularly MSCs play an integral role in dealing with cardiac diseases since they regenerate damaged cardiac tissue by being differentiated into cardiomyocytes. ${ }^{6}$ MSCs-cardiomyocytes differentiation is stimulated through the alteration in the cellular pattern of genes expression mediated by early transcriptional regulators such as NKX2.5 and GATA4. ${ }^{7}$ The early transcriptional factors regulate differentiation-specific gene expression which program various aspects of cardiac cell; this is especially done by inducing the expression of the late myocardial markers such as lactate dehydrogenize (LDH) and cardiac troponin I (cTnI). ${ }^{7,8}$

The tissue source can determine the differentiation potential of MSCs that control their efficiency as cellular therapy of cardiac diseases. ${ }^{9}$ In the 1960s, MSCs were being isolated from bone marrow, ${ }^{10}$ that has now become the most common MSCs' source, depended upon in clinical trials. Also, adipose tissue is a well-known source of MSCs; its easy isolation by liposuction has greatly attracted the attention of researchers and clinical trials. ${ }^{1}$ Although bone marrow and adipose tissue are accessible and enriched sources of MSCs, the capacity of their isolated MSCs to differentiate into cardiomyocytes is not satisfying ${ }^{11}$ since other tissues can be proved to introduce a higher differentiation potential of MSCs.

Despite the pluripotency of embryonic stem cells, the ethical concerns limit its use in cell therapy. ${ }^{12}$ Human umbilical cord (HUC) is a fetal tissue arising from the extra-embryonic mesoderm at day-13 of embryogenesis. So its isolated MSCs could possess higher differentiation and stemness properties than other traditional adult sources. The stromal part of the HUC is known as Wharton's jelly (WJ) that is the least studied source of MSCs. HUC-WJ is a medical waste discarded after the birth of a baby, so it is free from any ethical issues. ${ }^{13}$ Beside their ethical isolation availability from HUC-WJ, MSCs can show a high cardiac differentiation potentiality.

Previously, studied protocols could neither introduce a sufficient way of isolating pure MSCs from HUM-WJ nor an effective way of in vitro MSCs differentiation into cardiomyocytes. ${ }^{14}$ Those studies depended on treating MSCs with specific growth factors through inhibiting DNA methyltransferase. 5-azacytidine (5-Aza) is a DNA methyltransferase inhibitor that has an important role in the epigenetic regulation of genome expression. ${ }^{15}$ Sufficient differentiation of MSCs requires a high dose of 5-Aza, but it becomes cytotoxic. ${ }^{16}$ Thus, we recommend a new protocol by supporting 5-Aza in the differentiation of MSCs without being toxic.

Consequently, the current study established an effective strategy for the isolation of pure MSCs from HUMWJ as a perfect source of stem cells. It also suggested a new protocol for the expansion and differentiation of HUM-WJ isolated MSCs into cardiomyocyte-like cells in vitro. 


\section{Materials and Methods}

Chemicals

Culture media including DMEM, FBS, l-glutamine, penicillin-streptomycin, and $0.05 \%$ trypsin-EDTA were used in culturing MSCs. Cell culture medium and collagenase-A were obtained from Miltenyi Biotec, Bergisch Gladbach, Germany. Basic fibroblast growth factor (BFGF) and 5-Aza was purchased from Sigma-Aldrich, St. Louis, MO, USA. Deionized water was used throughout the experiments.

\section{Sample Collection}

A tissue sample isolated from a HUC was acquired from the Department of Gynecology and Obstetrics, Mansoura University, Egypt, from one pregnant female (30 years old), immediately after delivery. This is done under IRB approved protocol (MZ16001), which was following the Declaration of Helsinki. Accordingly, an informed written consent was obtained from the patient. HUC was collected in a sterile conical vial containing DMEM supported by $5 \%$ penicillin-streptomycin. The sample delivery to the laboratory as well as all manipulation were carried out under sterile conditions. The sample was delivered to the culture laboratory and stored at $4{ }^{\circ} \mathrm{C}$ for $2 \mathrm{hr}$ before tissue processing.

\section{Isolation and Establishment of the Primary HUC-WJ Isolated MSC Line}

The whole HUC was washed in sterile phosphate buffer saline (PBS) triplicate to remove any red blood cells. A small piece about 2-3 cm from the HUC was placed in a petri dish, following by removing blood vessels then the WJ was carefully separated from the HUC membrane. WJ was cut into small fragments with sharp scissor scalpels. After WJ has been minced into $1-2 \mathrm{~mm}^{3}$ fragments, the fragments were treated with collagenase-A ( $3 \mathrm{mg} / \mathrm{mL})$ in a $50 \mathrm{~mL}$ falcon tube for $3 \mathrm{hr}$ at $37^{\circ} \mathrm{C}$ in a shaking water bath $(125 \mathrm{rpm})$. Then the slurry was centrifuged for $15 \mathrm{~min}$ at $1500 \mathrm{xg}$ at $4^{\circ} \mathrm{C}$. The supernatant was subsequently discarded and the remaining slurry was collected to be treated with trypsin-EDTA for 30 minutes at $37^{\circ} \mathrm{C}$ in $5 \%$ $\mathrm{CO}_{2}$ with a humidified atmosphere. The cells were filtered with sterile $70 \mu \mathrm{L}$ sieve and were consequently re-suspended in DMEM, centrifuged at $1500 \times \mathrm{g}$ for 10 minutes. DMEM was aspirated, and the cell pellet was collected.

\section{Cell Culture}

The primary HUM-WJ isolated cell line was cultured by DMEM supplemented with 10\% FBS, 1\% penicillin-streptomycin, and 1\% l-glutamine; stored at $4{ }^{\circ} \mathrm{C}$ and warmed to $37{ }^{\circ} \mathrm{C}$ before using. This is followed by incubation in a $37{ }^{\circ} \mathrm{C}, 5 \% \mathrm{CO}_{2}$ incubator. The cells were fed with fresh medium every 2-3 days after aspirating the old medium. A few days later, colonies of fibroblast cells were seen. After reaching 80\% confluence, cells were trypsinized and sub-cultured in fresh supplemented DMEM into two new culture flasks to allow more space for further proliferation. Detached cells before each new passage were counted and tested for viability with trypan blue dye exclusion assay.

\section{Characterization of MSCs}

The mesenchymal characteristic of the cultured cells was analyzed by flow cytometry. At the 3rd and 4th passages, cells were detached and washed twice with PBS. Then, cells were mixed with fluorescein isothiocyanate (FITC) monoclonal-antibodies against human CD90, CD105, CD106, CD45, and CD146. All antibodies were purchased from BD Biosciences, San Jose, CA, USA. Mixed cells were incubated on ice in the dark for 15 minutes, washed with PBS, and suspended $\left(5 \times 10^{5}\right.$ cells $)$ in $1 \mathrm{~mL}$ PBS. Expressions of these markers was measured with a flow cytometer (FACS Aria Cell Sorter; BD Biosciences, Billerica, MA, USA). An appropriate isotype-matched control was used in all analysis, and unstained MSCs were used as control.

\section{Cardiac Induction}

In the 5 th passage, the $80 \%$ confluent cultured MSCs were divided into three cell groups. G1 (undifferentiated MSCs) was fed with complete medium and refreshed every $72 \mathrm{hr}$. This cell line was used as a control for the differentiated cells. G2 was fed with culture media supported only by 15 $\mu \mathrm{M}$ 5-Aza, while G3 was fed with culture media supported by $15 \mu \mathrm{M} 5$-Aza and $10 \mathrm{ng} / \mathrm{mL}$ BFGF. The differentiated cells (G2 and G3) were incubated with their differentiation media for $24 \mathrm{hr}$ and were then washed by PBS twice. Then, the cardiac induction media was replaced with a complete medium that replaced every $72 \mathrm{hr}$ during the differentiation period (three weeks). 


\section{Characterization of Cardiac}

\section{Differentiation Efficiency}

Ultra-Structural Analysis

After the period of cardiac differentiation induction, ultrastructural analysis with transmission electron microscopy (TEM) was performed on the tested cell groups. The collecting cell pellet was centrifuged at $1200 \mathrm{rpm}$ for 10 min and was then washed in $2 \%$ sodium phosphate buffer osmium tetroxide $(\mathrm{pH}=7.4)$ triplicate for $30 \mathrm{~min}$. The initial fixation was done in sodium phosphate buffer containing glutaraldehyde and 2\% paraformaldehyde. The dehydration was done with $50 \%$ ethanol for $9 \mathrm{~min}$. This is followed by washing the cells with distilled water twice for $15 \mathrm{~min}$. The cells were later embedded in epoxy resin. Ultrathin sections were cut and observed at $80 \mathrm{kV}$ using a JEOL 2100 TEM (JEOL GmbH, Eching, Germany).

\section{Quantification Assays for Cardiac Markers}

After the period of cardiac differentiation, the cell culture supernatant was collected from differentiated and undifferentiated cells. Any debris was removed from the supernatant media by centrifuging for $10 \mathrm{~min}$ at $2000 \mathrm{xg}$. The clear supernatant was used to analyze two cardiac markers: $\mathrm{LDH}$ and cTnI, triplicate for each cell group. According to the manufacturer's instructions, LDH and cTnI were measured with a commercial kit (Elitech, France) and spectrophotometer.

\section{Gene Expression Analysis}

The traditional PCR and the real-time qPCR (RT-qPCR) analysis were applied to RNA that was isolated from the differentiated and undifferentiated cells by using the TRIzol reagent and purified with GeneJET ${ }^{\mathrm{TM}}$ RNA Purification Kit (Fermentas Thermo Fisher Scientific). The RNA sample was diluted in DEPC water before it was quantified by spectrophotometry at $260 \mathrm{~nm}$. RNA ( $2 \mu \mathrm{g})$ from each cell group was applied for reverse transcription using the Maxima ${ }^{\circledR}$ First Strand cDNA Synthesis Kit (Fermentas Thermo Fisher Scientific). RT-qPCR was applied with the Maxima ${ }^{\circledR}$ SYBR Green qPCR Master Mix (Fermentas Thermo Fisher Scientific). Reactions were done in a $20 \mu \mathrm{L}$ volume. The primers for the TNNT1, NKX2.5, and DES genes are shown in the Supplementary material S1, with GAPDH as a control. qPCR was performed in triplicate for each sample with a RT-PCR detection system (Agilent Technologies) with an initial denaturation at $95^{\circ} \mathrm{C}$ for 10 min, followed by 40 cycles for $15 \mathrm{~s}$ at $95^{\circ} \mathrm{C}$, the $30 \mathrm{~s}$ at melting temperature, $5^{\circ} \mathrm{C}$, and $30 \mathrm{~s}$ at $72^{\circ} \mathrm{C}$. Specificity was detected with melting curve analysis. RT-qPCR products were electrophoresed on (1-2)\% agarose gels (according to the size of the amplification products). The gene expression levels of differentiated and undifferentiated cells were investigated with the comparative CT method. The observations were performed with the Real-Time PCR System (Applied Biosystems).

\section{Statistical Analysis}

All data were expressed as means \pm S.E. statistical significance and were evaluated by one-way analysis of variance (ANOVA) using SPSS, 18.0 software, 2011, the individual comparisons were obtained by Duncan's multiple range test (DMRT). Values were considered statistically significant when $\mathrm{P} \leq 0.05$.

\section{Results}

\section{Morphological Characteristics of HUM-WJ Isolated MSCs}

Freshly isolated HUM-WJ isolated MSCs appeared as heterogeneous sphere-shaped cells in suspended media under an inverted microscope (Figure 1A). After $72 \mathrm{hr}$ in primary culture, MSCs adhered to the plastic surface forming small clusters of swirl-like cells and spreading on the plate (Figure 1B). During early culture, MSCs proliferated gradually and rapidly until they covered the culture plate, and their morphology changed to be atypical aggregated fibroblasts. In the 3rd passage, MSCs had a clear slender, elongated, spindle shape with extended processes (Figure 1C).

\section{Flow Cytometry Analysis}

Flow cytometric analysis was performed on HUM-WJ isolated MSCs using antibodies against CD106, CD105, CD90, CD45, and CD146 cell surface markers. The result showed that the tested cells were positive for MSCs markers, such as CD106 (Figure 2A), CD105 (Figure 2B), and CD90 (Figure 2C), which were highly expressed by $87.8 \%$, $60.8 \%$, and $47.6 \%$, respectively in tested cells. On the other hand, tested cells were negative for HSCs markers, such as CD45 (Figure 2D) and CD146 (Figure 2E), which had a low expression of $1.8 \%$ and $2.2 \%$, respectively.

\section{Morphological Characteristics of Differentiated Cells}

After $24 \mathrm{hr}$ of incubation with differentiated media, the viability of HUM-WJ isolated MSCs in GII, that is affected 

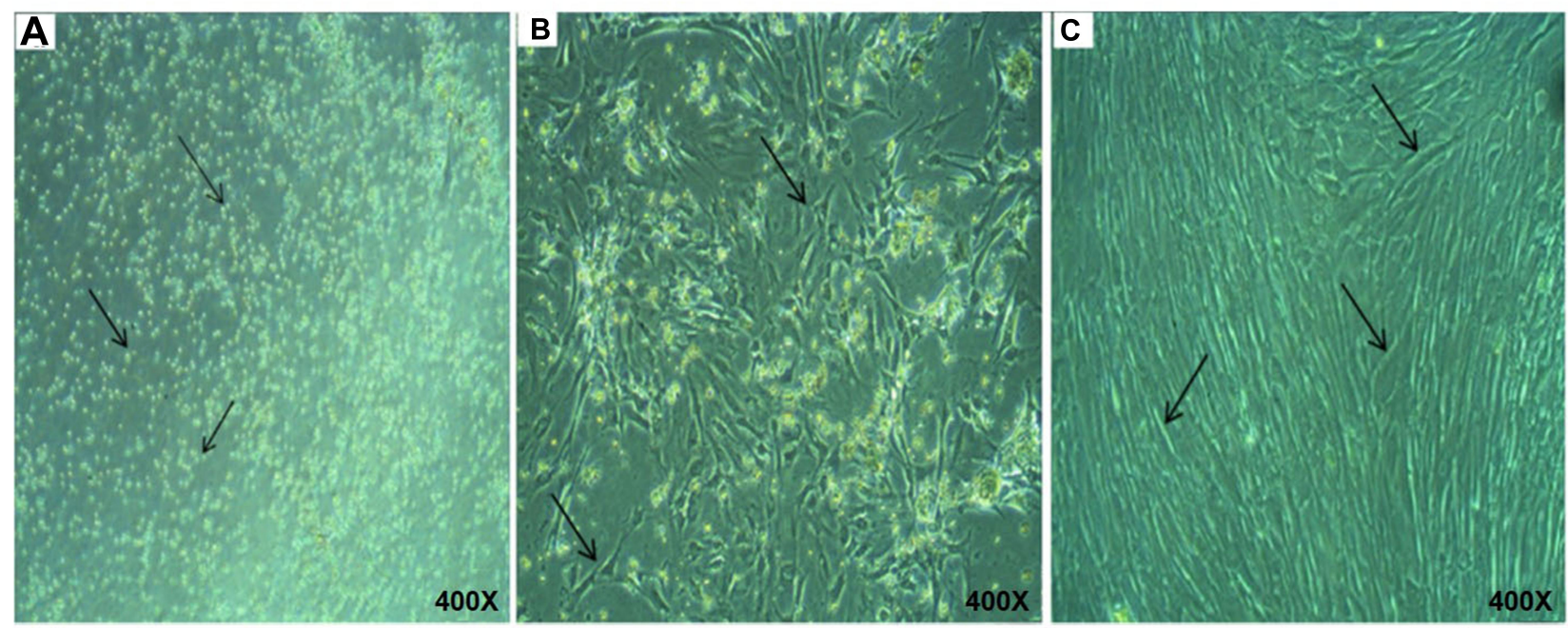

Figure I Morphological characteristics of HUM-WJ isolated MSCs.

Note: (A) Morphology of HUM-WJ isolated MSCs after $24 \mathrm{hr}$ in culture; arrows point to the spherical shape of suspended cells, (B) after 72 hr in culture; arrows point to the swirl-like cells, and (C) after 4 weeks in culture; arrows point to the spindled shaped cells.

Abbreviations: HUM-WJ, human umbilical cord-Wharton's jelly; MSCs, mesenchymal stem cells.
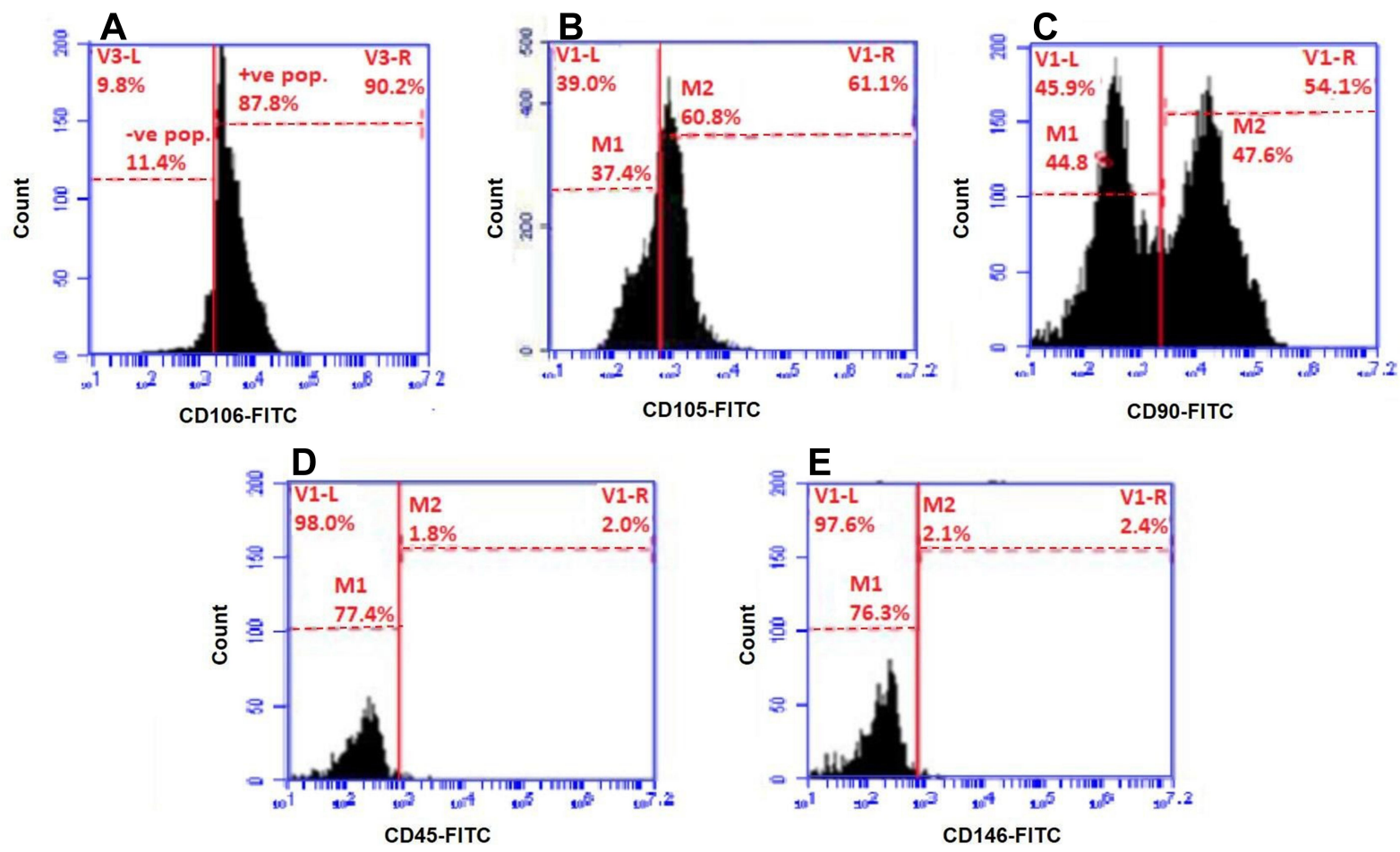

Figure 2 Flow cytometry analysis of HUM-W] isolated MSCs.

Note: Flow cytometry analysis of HUM-WJ isolated MSCs; (A) with CDI06, (B) with CDI05, (C) with CD90, (D) with CD45, and (E) with CDI46 markers. Abbreviations: HUM-WJ, human umbilical cord-Wharton's jelly; MSCs, mesenchymal stem cells.

by differentiated media, was visually observed (Figure 3A). On the other hand, compared with GII, differentiated media in GIII had a limited effect on cellular viability (Figure 3B).
The viable MSCs in both groups proliferated and differentiated gradually throughout the days that followed induction. One week later, the morphology of GII cells changed by 
expanding and enlarging (Figure 3C), like the morphology of GIII cells. However, GIII cells showed more noticeable expansion (Figure 3D). Three weeks later, cells in GII became elongated and aligned forming a stick- like morphology with extensions linked to the surrounding cells (Figure 3E). However, cells in GIII, as visually observed, were typically differentiated with more regular uniformed morphology than those of GII (Figure 3F). These visual observations were detected by an inverted microscope by making a comparison with cells in GI, preserved with their original spindled fibroblastic morphology during culture, as a control.

\section{Ultrastructural Characterization of the Differentiated Cells}

TEM observation of MSCs in GI was photographed to be a controlling factor for the differentiated cells, which were detected with the absence of any myofilaments (Figure 4A). TEM observation of cells in GII and GIII, after three weeks of induction, generally showed the differentiated cells with a centric nucleus and richer organelles in the cytoplasm, as rough endoplasmic reticulum, mitochondria, and free ribosomes. Specifically, cells in GII showed abundant myofilaments that aligned in a parallel shape without the appearance of striated sarcomeres (Figure 4B). Also, cells in GIII showed myofilaments but with extra typical striated sarcomeres and a denser look of granulated cytoplasm (Figure 4C).

\section{Quantification Assays for Cardiac Markers}

The protein expressions of cardiac markers (LDH and cTnI) were measured in media and they revealed the functionality of the differentiated cells' dependent manner. The results showed that differentiated cells of both GII and GIII expressed significantly higher levels of LDH (Figure 5A) and cTnI (Figure 5B) proteins, compared to their corresponding undifferentiated cells in GI $(\mathrm{P}<0.05)$. However, these expressions increased by the time of induction. Limited expression of cardiac markers was specifically detected in cells of GII compared to cells of GIII that showed a remarkable higher expression of these markers.

\section{Molecular Analysis}

The traditional PCR analysis (Figure 6A) and the RTqPCR analysis (Figure 6B) were carried out to measure the fold changes in the expression of NKX2.5, TNNT1, and Desmin by differentiated cells in GII and GIII, in comparison with cells of GI as a control. Cells of GII and GIII actively expressed NKX2.5, especially after
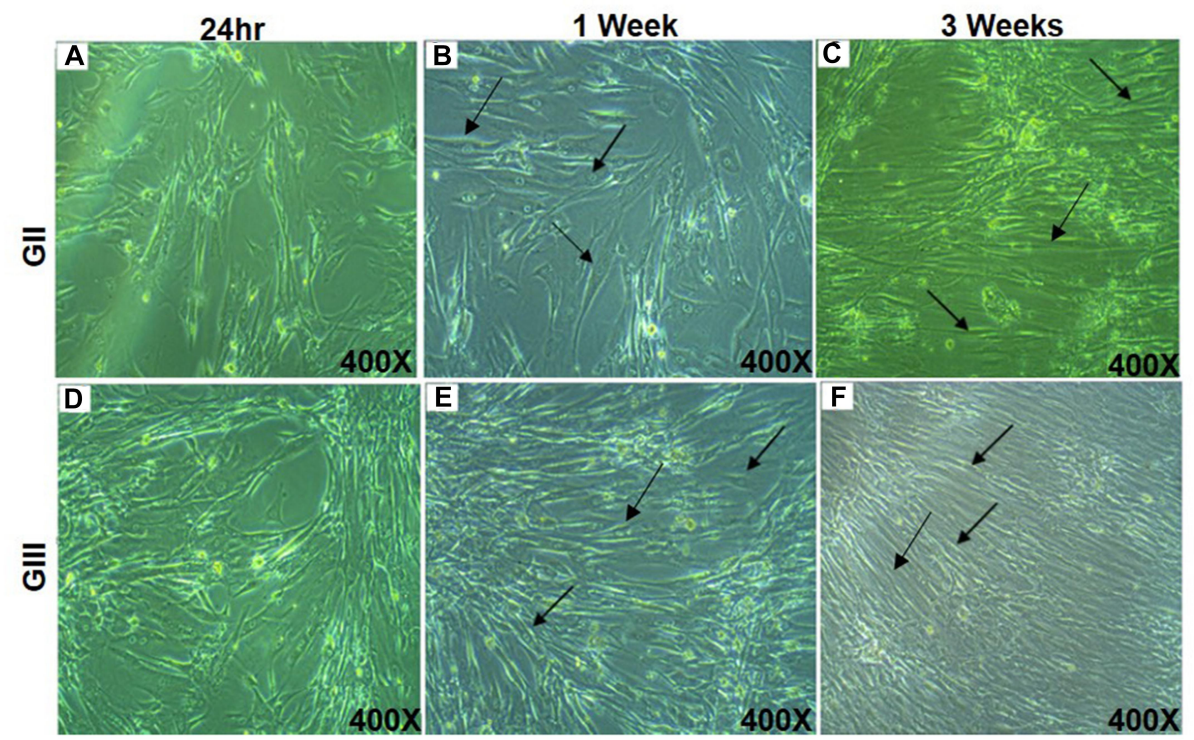

Figure 3 Morphological characteristics of differentiated cells.

Note: Morphological changes were examined by inverted microscope in cells of (A) Gll after 24 hr, (B) GIII after 24 hr, (C) GIl after I week; arrows point to expanding cells, (D) GIII after I week; arrows point to large expansion of cells, (E) GIl after 3 weeks; arrows point to cells with stick like morphology, and (F) GIII after 3 weeks of induction; arrows point to uniform stick like morphology of cells. 

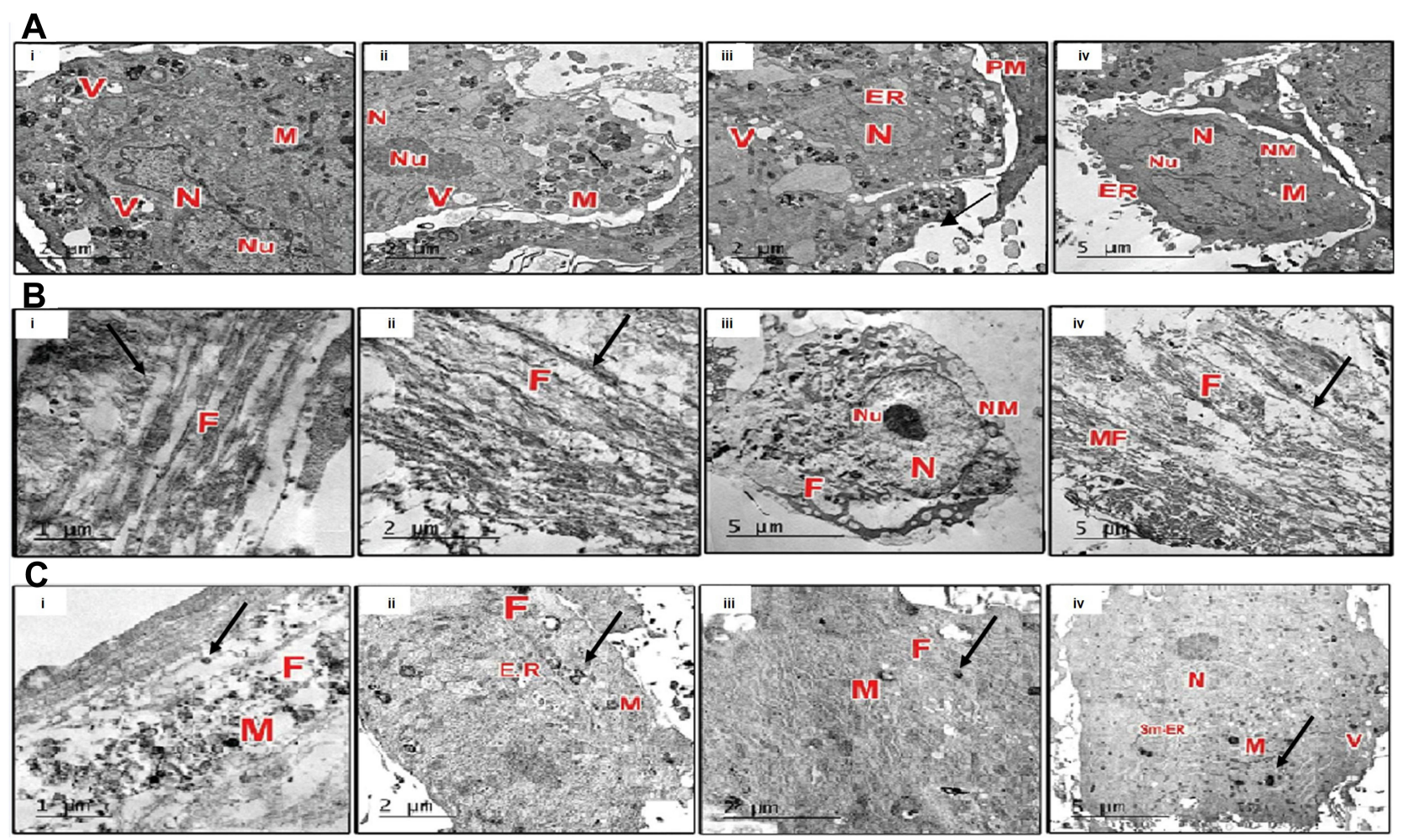

Figure 4 Ultrastructural characterization of the differentiated cells.

Note: Ultrastructural changes was examined by TEM in cells of (A; i, ii, iii, iv) Gl as a control, (B; i, ii, iii, iv) Gll; arrows point to abundant aligned myofilaments, and (C; i, ii, iii, iv) GIII; arrows point to aligned myofilaments with striated sarcomeres.

Abbreviations: N, nucleus; $\mathrm{Nu}$, nucleolus; NM, nuclear membrane; ER, endoplasmic reticulum; Sm-ER, smooth endoplasmic reticulum; PM, plasma membrane; M, mitochondria; MF, myofilaments; F, fibrils; and V, vacuoles.

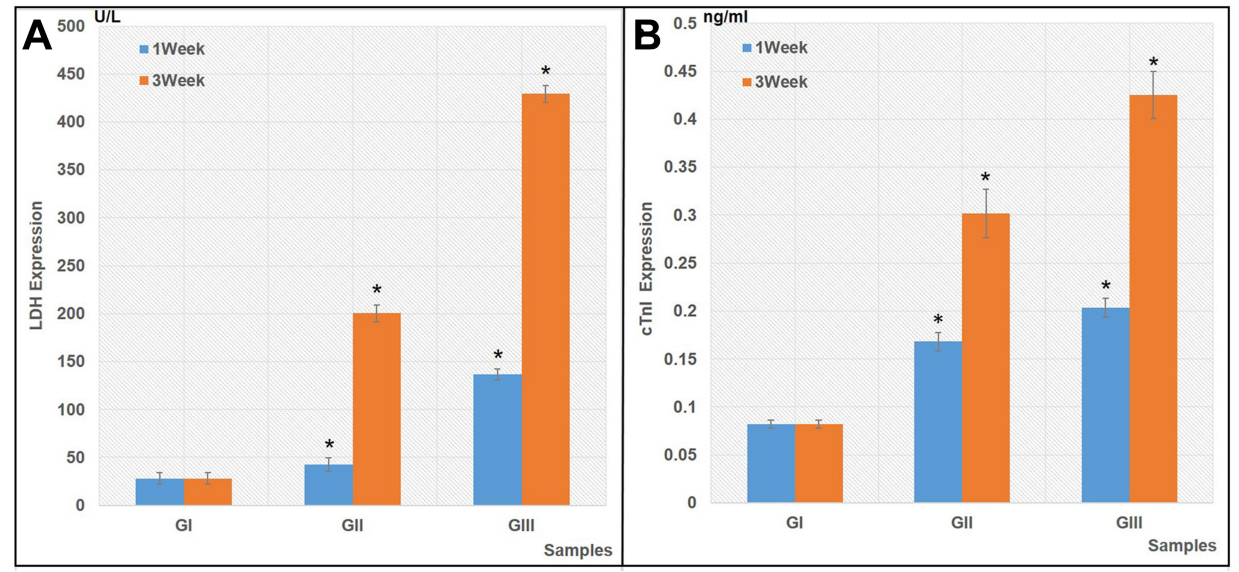

Figure 5 Quantification expressions for cardiac markers.

Note: Quantitative expressions for (A) LDH marker, and (B) cTnl marker in differentiated cells, *P<0.05 vs Control.

Abbreviations: $\mathrm{LDH}$, lactate dehydrogenize; and $\mathrm{cTnl}$, cardiac troponin I.

one week with 10.97 -fold and $4.40-$ fold, respectively, which are significantly higher than GI $(\mathrm{P}<0.05)$ (Figure 6B.i). Also, TNN1 gene was highly expressed by cells of GII and GIII, especially after three weeks with 3.54-fold and 2.64-fold, respectively, which are significantly higher than cells of GI $(\mathrm{P}<0.05)$ (Figure 6B.ii). Moreover, cells of GIII highly expressed Desmin, especially after three weeks with 2.36-fold, 


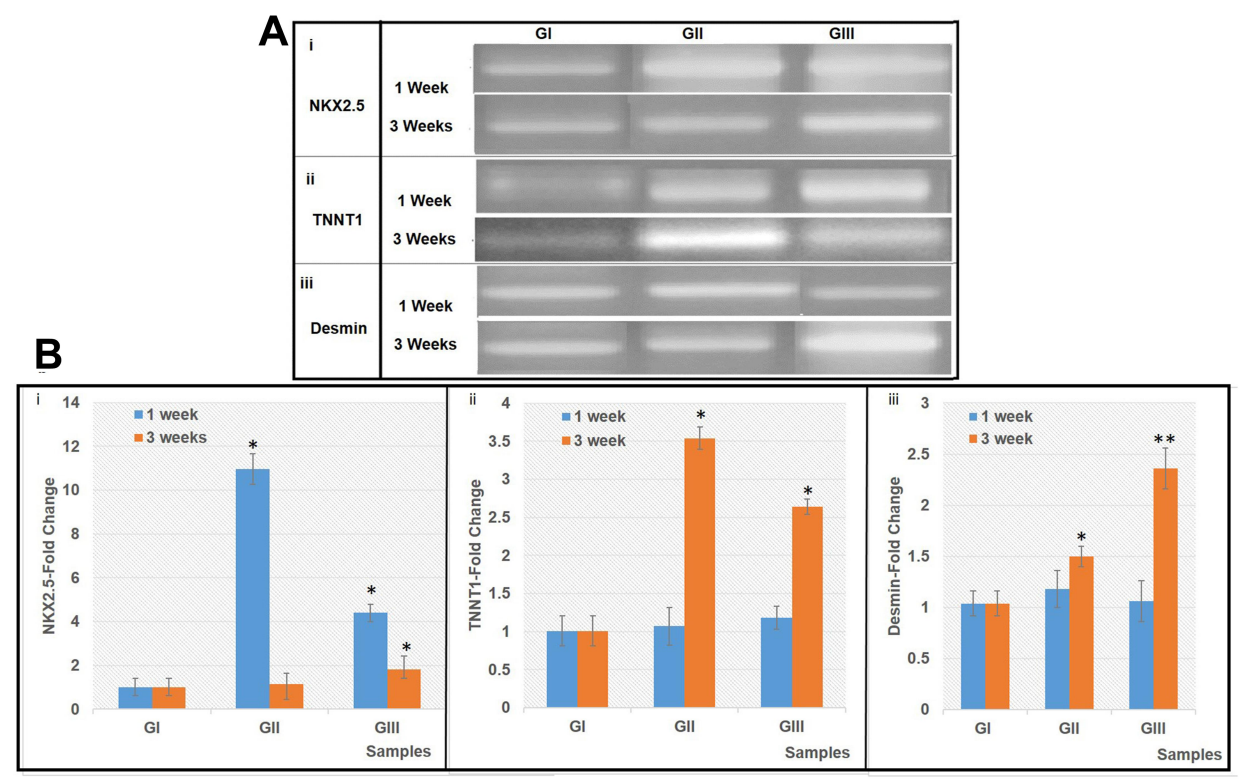

Figure 6 Expression of NKX2.5, TNNTI and Desmin mRNA.

Notes: (A) PCR analysis for gene expression and (B) RT-qPCR analysis for fold change of gene expression. (I) NKX2.5, (II) TNNTI and (III) Desmin. Fold change of GAPDH was used as an internal control. *P $<0.05$ vs Control, and $* * \mathrm{P}<0.05$ vs Control and Gll.

which is significantly higher than the other differentiated cells in GII and control cells in GI $(\mathrm{P}<0.05)$ (Figure 6B.iii).

\section{Discussion}

Recently, regenerative medicine based on MSCs becomes a promising therapeutic strategy for many diseases such as cardiac diseases. ${ }^{18}$ Many previous studies focused on wellknown tissues as sources for MSCs such as; bone marrow and adipose tissue. ${ }^{19}$ Although bone marrow isolated extracted MSCs proved their efficiency in the treatment of many diseases, such as orthopedic disorders, this is related to their expression of genes associated with the osteogenetic habit. ${ }^{20}$ Thus, bone marrow isolated MSCs are not the best choice in the treatment of other diseases such as cardiac diseases. This study tested the hypothesis that: HUC-WJ is an unusual MSCs source. Not only for its ethically legal, but also for its higher differentiation potential. Therefore, we focused on HUC-WJ as a challenging ethical fetal tissue that is better than other conventional adult tissues as a competitive source for MSCs with cardiac differentiation potency.

In the present study, primary HUC-WJ isolated MSCs line was established by the formulated simple method depending upon enzymatic digestion. It does not perform only by collagenase, but also by the effect of trypsin to ensure a sufficient count of MSCs. ${ }^{21}$ The purity of primary cultured adherent cells with their microscopic spindle appearance is confirmed by flow cytometry, depending on previous studies. It is estimated that MSCs were expressed by specific markers such as CD106, $\mathrm{CD} 105$, and CD90. ${ }^{22} \mathrm{CD} 106$ is a member of the immunoglobulin superfamily and it was highly expressed in these cells $(87.8 \%)$. It is also known as vascular cell adhesion molecule-1 (VCAM-1), because it has an essential role in adhesion and homing. ${ }^{23} \mathrm{CD} 105$ or endoglin was expressed in $60.8 \%$ of tested cells whereas it is necessary for cell division and cellular differentiation. ${ }^{24}$ An extra MSCs specific marker, CD90 or Thy- 1 is a cell surface anchored glycoprotein, and it is essential for cell mobility and migration. ${ }^{25}$ It was positively expressed in $47.6 \%$ of the tested cells. Many previous studies estimated that MSCs were negative for the hematopoietic marker (CD45) and endothelial marker (CD146). ${ }^{5}$ Since CD45 is a transmembrane protein tyrosine phosphatase that controls the motility of hematopoietic progenitor cells and the maturation of leukocytes as lymphocytes. ${ }^{26}$ Besides, CD146 or M-CAM regulates the migration and motility of endothelial cells. ${ }^{27}$ In the present study, CD $45^{-\mathrm{ve}}$ and $\mathrm{CD} 146^{-\mathrm{ve}}$ (1.8\% and $2.2 \%$; respectively) proved the purity of the mesenchymal nature of tested cells and the lacking of contamination with other cell types.

The study also tested the differentiation of HUC-WJ isolated MSCs into cardiomyocytes that were enhanced, not only by a 5 -Aza factor but also by 5-Aza with BFGF. We hypothesized the combination of 5-Aza and BFGF as having a synergic effect in the differentiation of MSCs into cardiomyocytes. Previous studies concluded that many growth factors can 
stimulate higher myocardial differentiation of MSCs and induce the gene expression related to cardiomyocytes under proper condition. ${ }^{28,29}$ Although 5-Aza is known as a differential factor for MSCs into cardiomyocytes cells, ${ }^{30,31}$ it could not be regarded as safe and sufficient for inducing the cardiomyogenic fate. ${ }^{32,33} \mathrm{BFGF}$ is considered a potential coinducer required to support the effect of 5-Aza in forcing the differentiation of MSCs into cardiomyocytes. ${ }^{34}$ In the current study, the morphological changes of MSCs confirmed this hypothesis: 5-Aza affect harmfully the viability of cells that were handled by BFGF. The differentiation of MSCs was improved by BFGF that supported the effect of 5-Aza through the remarkable morphological expansion of differentiated cells. The existence of primitive myofilaments was the sharp morphological sign that emphasized the higher differentiation induction effect of the 5-Aza and BFGF combination.

The present study showed the morphological changes in MSCs resulted from the expression of cardiomyogenic proteins. Whereas, cell culture supernatant showed a high level of LDH and cTnI significantly after cellular differentiation. LDH is mainly on the filaments of cardiac muscle fibers that have immune-function. cTnI is a part of the contractile mechanism of the cardiac muscle. ${ }^{35}$ The synergic effect of both myocardial differential inducers (5-Aza and BFGF) of MSCs is confirmed through the upregulation of these cardiac-specific biomarkers by time. This result confirmed that cardiogenic differentiation is performed not only by $5-\mathrm{Aza}^{36}$ but also by the help of BFGF in regulating the myocardial differentiation. ${ }^{37}$

5-Aza induced MSCs were previously proved to be able to express cardiac-specific genes such as NKX2.5, TNNT1, and Desmin, ${ }^{38}$ while every gene has its specific cardiogenic role. The study also highlighted NKX2.5 or homeobox gene as the earliest-specific marker of cardiac lineage during embryogenesis. ${ }^{39}$ It also shows how TNNT1 gene encodes the slow skeletal muscle troponin. ${ }^{40}$ Desmin is an essential gene in maintaining the structure of sarcomeres as it has a necessary role in cardiac muscle tone. ${ }^{41}$ Thus, this study detected the progress of myocardial differentiation of MSCs through the expression of these genes at the RNA level to determine the best differential protocol in a time-dependent manner (one and three weeks). Both protocols of differentiation confirmed a significantly higher expression of these cardiac-specific genes in comparison with undifferentiated cells. TNNT1 and Desmin expression increased gradually by time, while NKX2.5 is an early marker that elevated in the first week and peaked after three weeks from the starting of induction in both protocols. Particularly the significantly high level of
Desmin was detected after differential induction by extra help of BFGF. The expression of these cardiac genes encoded the morphological and functional properties of differentiated cells. ${ }^{42}$ So high expression of TNNT1, Desmin, and NKX2.5 was driving the cardiomyocytes' features and the expression of functional proteins. The results showed the cardio-differential potency of HUC isolated MSCs through the morphological, the functional, and the genetic levels.

Collectively, the results of this study recommend using HUC-WJ as a competitive source of MSCs, that has a promising regenerative therapeutic effect on cardiac diseases. Significant differentiation of HUC-WJ isolated MSCs into cardiomyocyte-like cells has been detected in vitro by combining 5-Aza with BFGF. In addition, in vivo experimental studies and functional tests are requested for the myocardial differentiation of HUC-WJ isolated MSCs in order to be applicable in the clinical trials.

\section{Conclusion}

In brief, this study concluded applying for regenerative medicine by MSCs may represent a therapeutic strategy for achieving better treatment of cardiac diseases. The study presents HUM-WJ as a competitive source for seeding MSCs, and an efficient mechanism mediated by 5-Aza and BFGF to enhance the HUC-WJ isolated MSCs with myocardial features.

\section{Acknowledgments}

We thank "MERC" of Mansoura Faculty of Medicine for their support. We appreciate the excellent English editorial assistance of Nermine A. Gomaa, associate professor of English literature at Horus University.

\section{Disclosure}

The authors report no conflicts of interest in this work.

\section{References}

1. Karantalis V, Hare JM. Use of mesenchymal stem cells for therapy of cardiac disease. Circ Res. 2015;116(8):1413-1430.

2. Roura S, Gálvez-Montón C, Mirabel C, Vives J, Bayes-Genis A. Mesenchymal stem cells for cardiac repair: are the actors ready for the clinical scenario? Stem Cell Res Ther. 2017;8(1):238. doi:10.1186/s13287-017-0695-y

3. Majka M, Sułkowski M, Badyra B, Musiałek P. Concise review: mesenchymal stem cells in cardiovascular regeneration: emerging research directions and clinical applications. Stem Cells Transl Med. 2017;6(10):1859-1867. doi:10.1002/sctm.16-0484

4. Ullah I, Subbarao RB, Rho GJ. Human mesenchymal stem cells current trends and future prospective. Biosci Rep. 2015;35(2):e00191. doi:10.1042/BSR20150025

5. Maleki M, Ghanbarvand F, Reza Behvarz M, Ejtemaei M, Ghadirkhomi E. Comparison of mesenchymal stem cell markers in multiple human adult stem cells. Int J Stem Cells. 2014;7(2):118-126. doi:10.15283/ijsc.2014.7.2.118 
6. Alrefai MT, Murali D, Paul A, Connell JM, Shum-Tim D. Cardiac tissue engineering and regeneration using cell-based therapy. Stem Cells Cloning. 2015;8:81-101.

7. Oka T, Xu J, Molkentin JD. Re-employment of developmental transcription factors in adult heart disease. Semin Cell Dev Biol. 2007;18 (1):117-131. doi:10.1016/j.semcdb.2006.11.012

8. Singh A, Singh A, Sen D. Mesenchymal stem cells in cardiac regeneration: a detailed progress report of the last 6 years (20102015). Stem Cell Res Ther. 2016;7(1):82.

9. Xu L, Liu Y, Sun Y, et al. Tissue source determines the differentiation potentials of mesenchymal stem cells: a comparative study of human mesenchymal stem cells from bone marrow and adipose tissue. Stem Cell Res Ther. 2017;8(1):275. doi:10.1186/s13287-017-0716-x

10. Drzewiecki BA, Thomas JC, Tanaka ST. Bone marrow-derived mesenchymal stem cells: current and Future applications in the urinary bladder. Stem Cells Int. 2011;2010:765167.

11. Sun Q, Zhang Z, Sun Z. The potential and challenges of using stem cells for cardiovascular repair and regeneration. Genes Dis. 2014;1 (1):113-119.

12. Lo B, Parham L. Ethical issues in stem cell research. Endocr Rev. 2009;30(3):204-213. doi:10.1210/er.2008-0031

13. Kim DW, Staples M, Shinozuka K, Pantcheva P, Kang SD, Borlongan CV. Wharton's jelly-derived mesenchymal stem cells: phenotypic characterization and optimizing their therapeutic potential for clinical applications. Int J Mol Sci. 2013;14(6):11692-11712. doi:10.3390/ijms 140611692

14. Meng M, Liu Y, Wang W, et al. Umbilical cord mesenchymal stem cell transplantation in the treatment of multiple sclerosis. Am J Transl Res. 2018;10(1):212-223.

15. Secco M, Zucconi E, Vieira NM, et al. Multipotent stem cells from umbilical cord: cord is richer than blood. Stem Cells. 2008;26 (1):146-150. doi:10.1634/stemcells.2007-0381

16. Lee S, Kim HS, Roh KH, et al. DNA methyltransferase inhibition accelerates the immunomodulation and migration of human mesenchymal stem cells. Sci Rep. 2015;5:8020. doi:10.1038/srep08020

17. Wang HS, Hung SC, Peng ST, et al. Mesenchymal stem cells in the Wharton's jelly of the human umbilical cord. Stem Cells. 2004;22:1330-1337. doi:10.1634/stemcells.2004-0013

18. Weiss ML, Medicetty S, Bledsoe AR, et al. Human umbilical cord matrix stem cells: preliminary characterization and effect of transplantation in a rodent model of Parkinson's disease. Stem Cells. 2006;24:781-792. doi:10.1634/stemcells.2005-0330

19. Abbas OL, Özatik O, Gönen ZB, et al. Comparative analysis of mesenchymal stem cells from bone marrow, adipose tissue, and dental pulp as sources of cell therapy for zone of stasis burns. J Invest Surg. 2018;1-14.

20. Panepucci RA, Siufi JL, Silva WA, et al. Comparison of gene expression of umbilical cord vein and bone marrow-derived mesenchymal stem cells. Stem Cells. 2004;22:1263-1278. doi:10.1634/stemcells.2004-0024

21. Salehinejad P, Alitheen NB, Ali AM. Comparison of different methods for the isolation of mesenchymal stem cells from human umbilical cord Wharton's jelly. In Vitro Cell Dev Biol Anim. 2012;48 (2):75-83. doi:10.1007/s11626-011-9480-x

22. Rojewski MT, Weber BM, Schrezenmeier H. Phenotypic characterization of mesenchymal stem cells from various tissues. Transfus Med Hemother. 2008;35(3):168-184. doi:10.1159/000129013

23. Yang ZX, Han ZB, Ji YR, et al. CD106 identifies a subpopulation of mesenchymal stem cells with unique immunomodulatory properties. PLoS One. 2013;8(3):e59354. doi:10.1371/journal.pone.0059354

24. Aslan H, Zilberman Y, Kandel L, et al. Osteogenic differentiation of noncultured immunoisolated bone marrow-derived $\mathrm{CD}_{105^{+}}$cells. Stem Cells. 2006;24:1728-1737.
25. Rege TA, Hagood JS. Thy-1 as a regulator of cell-cell and cell-matrix interactions in axon regeneration, apoptosis, adhesion, migration, cancer, and fibrosis. FASEB J. 2006;20:1045-1054. doi:10.1096/fj.05-5460rev

26. Shivtiel S, Kollet O, Lapid K, et al. CD45 regulates retention, motility, and numbers of hematopoietic progenitors, and affects osteoclast remodeling of metaphyseal trabecules. J Exp Med. 2008;205:2381-2395. doi:10.1084/jem.20080072

27. Ouhtit A, Gaur RL, Abd Elmageed ZY, et al. Towards understanding the mode of action of the multifaceted cell adhesion receptor CD146. Biochim Biophys Acta. 2009;1795:130-136.

28. Muguruma $\mathrm{Y}$, Reyes M, Nakamura $\mathrm{Y}$, et al. In vivo and in vitro differentiation of myocytes from human bone marrow-derived multipotent progenitor cells. Exp Hematol. 2003;31:1323-1330. doi:10.1016/j.exphem.2003.09.003

29. Bartunek J, Croissant JD, Wijns W, et al. Pretreatment of adult bone marrow mesenchymal stem cells with cardiomyogenic growth factors and repair of the chronically infarcted myocardium. Am J Physiol Heart Circ Physiol. 2007;292:1095-1104. doi:10.1152/ajpheart.01009.2005

30. Nartprayut K, U-Pratya Y, Kheolamai P, et al. Cardiomyocyte differentiation of perinatally-derived mesenchymal stem cells. Mol Med Rep. 2013;7:1465-1469. doi:10.3892/mmr.2013.1356

31. Li M, Ikehara S. Bone-marrow-derived mesenchymal stem cells for organ repair. Stem Cells Int. 2013;132642.

32. Bae S, Shim SH, Park CW, et al. Combined omics analysis identifies transmembrane 4 L6 family member 1 as a surface protein marker specific to human mesenchymal stem cells. Stem Cells Dev. 2011;20:197-203. doi:10.1089/scd.2010.0127

33. Tomita S, Li RK, Weisel R, et al. Autologous transplantation of bone marrow cells improves damaged heart function. Circulation. 1999;100(19):11247-11256. doi:10.1161/01.CIR.100.supp1_2.II-247

34. Heng BC, Haider HK, Sim EK, Cao T, Ng SC. Strategies for directing the differentiation of stem cells into the cardiomyogenic lineage in vitro. Cardiovasc Res. 2004;62:34 42. doi:10.1016/j.cardiores.2003.12.022

35. Brotto MA, Biesiadecki BJ, Brotto LS, Nosek TM, Jin JP. Coupled expression of troponin $\mathrm{T}$ and troponin I isoforms in single skeletal muscle fibers correlates with contractility. Am J Physiol Cell Physiol. 2006;290(2):567-576. doi:10.1152/ajpcell.00422.2005

36. Wan Safwani WK, Makpol S, Sathapan S, Chua KH. 5-Azacytidine is insufficient for cardiogenesis in human adipose-derived stem cells. J Negat Results Biomed. 2012;11:13. doi:10.1186/1477-5751-11-3

37. Rosenblatt-Velin N, Lepore MG, Cartoni C, Beermann F, Pedrazzini T. FGF2 controls the differentiation of resident cardiac precursors into functional cardiomyocytes. J Clin Invest. 2005;115 (7):1724-1733. doi:10.1172/JCI23418

38. Qian Q, Qian H, Zhang X, et al. 5-Azacytidine induces cardiac differentiation of human umbilical cord derived mesenchymal stemcells by activating extracellular regulated kinase. Stem Cells Dev. 2012;21(1):67-75. doi:10.1089/scd.2010.0519

39. Lien CL, Wu C, Mercer B, et al. Control of early cardiac-specific transcription of Nkx2-5 by a GATA-dependent enhancer. Development. 1999;126(1):75-84.

40. Kuroda T, Yasuda S, Nakashima H, et al. Identification of a gene encoding slow skeletal muscle troponin $\mathrm{t}$ as a novel marker for immortalization of retinal pigment epithelial cells. Sci Rep. 2017;7 (1):8163. doi:10.1038/s41598-017-08014-w

41. Paulin D, Li Z. Desmin: a major intermediate filament protein essential for the structural integrity and function of muscle. Exp Cell Res. 2004;301(1):1-7. doi:10.1016/j.yexcr.2004.08.004

42. Nomura S, Satoh M, Fujita T, et al. Cardiomyocyte gene programs encoding morphological and functional signatures in cardiac hypertrophy and failure. Nat Commun. 2018;9(1):4435. doi:10.1038/ s41467-018-06639-7 


\section{Publish your work in this journal}

Stem Cells and Cloning: Advances and Applications is an international, peer-reviewed, open access journal. Areas of interest in established and emerging concepts in stem cell research include: Embryonic cell stems; Adult stem cells; Blastocysts; Cordblood stem cells; Stem cell transformation and culture; Therapeutic cloning; Umbilical cord blood and bone marrow cells; Laboratory,

animal and human therapeutic studies; Philosophical and ethical issues related to stem cell research. This journal is indexed on CAS. The manuscript management system is completely online and includes a very quick and fair peer-review system, which is al easy to use. Visit http://www.dovepress.com/testimonials.php to read real quotes from published authors. 